\title{
Novel neuroblastoma amplified sequence (NBAS) mutations in a Japanese boy with fever-triggered recurrent acute liver failure
}

\author{
Sahoko Ono ${ }^{1}$, Junko Matsuda ${ }^{1,2}$, Etsuko Watanabe², Hiroto Akaike', Hideto Teranishi', Ippei Miyata (1), \\ Takanobu Otomo ${ }^{1,2}$, Yoshito Sadahira ${ }^{3}$, Tatsuki Mizuochi ${ }^{4}$, Hironori Kusano ${ }^{5}$, Masayoshi Kage ${ }^{6}$, Hiroo Ueno ${ }^{7}$, \\ Kenichi Yoshida 7 , Yuichi Shiraishi ${ }^{8,9}$, Kenichi Chiba ${ }^{8,9}$, Hiroko Tanaka ${ }^{8,9}$, Satoru Miyano ${ }^{8,9}$, Seishi Ogawa7, \\ Yasuhide Hayashi ${ }^{10}$, Hirokazu Kanegane ${ }^{11}$ and Kazunobu Ouchi ${ }^{1}$
}

\begin{abstract}
Biallelic mutations in the neuroblastoma amplified sequence (NBAS) gene have been reported to cause two different clinical spectra: short stature with optic nerve atrophy and Pelger-Huët anomaly (SOPH) syndrome and infantile liver failure syndrome 2 (ILFS2). Here, we describe a case of a 3-year-old Japanese boy who presented with fever-triggered recurrent acute liver failure (ALF). The clinical characteristics were considerable elevation of liver enzymes, severe coagulopathy, and acute renal failure. In addition to the liver phenotype, he had short stature and Pelger-Huët anomaly in the peripheral granulocytes. Whole-exome and Sanger sequencing of the patient and his parents revealed that he carried novel compound heterozygous missense mutations in NBAS, c.1018G >C (p.Gly340Arg) and c.2674 G>T (p.Val892Phe). Both mutations affect evolutionarily conserved amino acid residues and are predicted to be highly damaging. Immunoblot analysis of the patient's skin fibroblasts showed a normal NBAS protein level but a reduced protein level of its interaction partner, p31, involved in Golgi-to-endoplasmic reticulum retrograde vesicular trafficking. We recommend NBAS gene analysis in children with unexplained fever-triggered recurrent ALF or liver dysfunction. Early antipyretic therapy may prevent further episodes of ALF.
\end{abstract}

\section{Introduction}

Acute liver failure (ALF) is a life-threatening emergency in childhood. Although $~ 25 \%$ of ALF cases are caused by inborn errors of metabolism, the underlying cause remains unknown in $\sim 50 \%$ of cases ${ }^{1-3}$. Recently, biallelic mutations in the neuroblastoma amplified sequence $(N B A S)$ gene were identified as a new cause of infantile liver failure syndrome-2 (ILFS2, OMIM 616483) ${ }^{4}$. ILFS2 is an autosomal recessive genetic disorder connected with recurrent episodes of ALF triggered by febrile infection. Previously, the homozygous NBAS mutation c.5741G $>$ A

Correspondence: Junko Matsuda (matsujun@med.kawasaki-m.ac.jp) ${ }^{1}$ Department of Pediatrics, Kawasaki Medical School, Okayama 701-0192, Japan ${ }^{2}$ Department of Pathophysiology and Metabolism, Kawasaki Medical School, Okayama 701-0192, Japan

Full list of author information is available at the end of the article.
(p.Arg1914His) was reported to be associated with a syndrome comprising short stature with optic nerve atrophy and Pelger-Huët anomaly (SOPH) syndrome, which occurs in the genetically isolated Yakut population (OMIM \#614800) $)^{5}$. Increasing evidence has indicated a phenotypic spectrum of NBAS mutation-based disease, from isolated fever-triggered recurrent ALF to a multisystemic disease with short stature, skeletal dysplasia, immunological abnormalities, and optic nerve atrophy resembling SOPH syndrome ${ }^{6-18}$. Because early antipyretic therapy and induction of anabolism have effectively ameliorated the course of ALF with the NBAS mutation, it is important to differentially diagnose NBAS mutation-based disease. Here, we describe a 3-year-old Japanese boy who presented with recurrent episodes of

(c) (i) Open Access This article is licensed under a Creative Commons Attribution 4.0 International License, which permits use, sharing, adaptation, distribution and reproduction BY in any medium or format, as long as you give appropriate credit to the original author(s) and the source, provide a link to the Creative Commons license, and indicate if changes were made. The images or other third party material in this article are included in the article's Creative Commons license, unless indicated otherwise in a credit line to the material. If material is not included in the article's Creative Commons license and your intended use is not permitted by statutory regulation or exceeds the permitted use, you will need to obtain permission directly from the copyright holder. To view a copy of this license, visit http://creativecommons.org/licenses/by/4.0/. 
ALF and carries novel compound heterozygous missense mutations in NBAS.

\section{Materials and methods Subjects}

After informed consent and the approval of the appropriate institutional ethics review board, peripheral blood samples were obtained from the patient, his parents, and his younger brother, and skin fibroblasts were obtained from the patient. Genomic DNA was extracted from peripheral blood leukocytes.

\section{Whole-exome sequencing}

Exomes of the patient and his parents were captured from the genomic DNA using the Agilent SureSelect Human All Exon V5 kit (Agilent Technologies, Santa Clara, CA, USA) and were sequenced (paired-end, $2 \times$ $124 \mathrm{bp}$ ) using the Illumina HiSeq 2500 (Illumina, San Diego, CA, USA). Read alignment to the human reference genome hg19 was performed with Burrows-Wheeler Aligner (http://bio-bwa.sourceforge.net/), and ANNOVER was used for annotation. Germline mutations were detected through our established pipeline, as previously reported (http://genomon.hgc.jp/exome/en/index.html) ${ }^{19}$. Population frequencies in the Human Genetic Variation Database (HGVD), db single-nucleotide polymorphisms (SNP) 131, the Integrative Japanese Genome Variation Database (iJGVD) ${ }^{20}$, and an in-house SNP database were used to filter common SNPs and to confirm the novelty of each mutation. Suspected pathogenic variations in genes known to be associated with ALF or present in accordance with autosomal recessive or X-linked modes of inheritance were identified as candidate genes.

\section{Sanger direct sequencing}

To validate the two NBAS variants detected in exons 12 and 24 by whole-exome sequencing, polymerase chain reaction (PCR)-direct sequencing was carried out using the genomic DNA of the patient, his parents, and his younger brother. PCR amplification was carried out using primers specific for NBAS exon 12, (forward 5'-TGGCACCTC TAAAGAGTGTCATT-3', reverse 5'-CTCACCCTTAA GAGAGTATCATTCTAA-3') and exon 24 (forward 5'-GA ATTAGATTCTGTACTGGAGACTTTT-3', reverse 5'-TC AAAGTGCATAGAAAATGCTTTA-3'). PCR conditions are available on request. The amplified products were sequenced using an ABI 3130xl DNA Analyzer (Thermo Fisher Scientific, Waltham, MA, USA), and NM_015909 was used as the NBAS reference sequence.

\section{In silico analysis}

To predict the protein-damaging effects of the suspected pathogenic variations in $N B A S$, three different software programs were used: Polyphen-2 (http:// genetics.bwh.harvard.edu/pph2/), Provean (http:// provean.jcvi.org/), and MutationTaster (http://www. mutationtaster.org/).

\section{Western blot analysis}

Patient and control fibroblasts were cultivated in minimum essential medium (MEM) supplemented with $10 \%$ fetal bovine serum (FBS) and $1 \%$ penicillinstreptomycin at 37 and $40{ }^{\circ} \mathrm{C}$, under $5 \% \mathrm{CO}_{2}$. For Western blots, cells were collected, washed in phosphate-buffered saline (PBS) and resolved in radioimmunoprecipitation assay (RIPA) buffer [50 $\mathrm{mM}$ tris(hydroxymethyl) aminomethane (Tris) base $\mathrm{pH} 7.5$, $150 \mathrm{mM} \mathrm{NaCl}, 1 \mathrm{mM}$ ethylenediaminetetraacetic acid (EDTA), 1\% NP-40, 0.5\% sodium deoxycholate, and $0.1 \%$ sodium dodecyl sulfate (SDS)] containing protease inhibitor cocktail tablets (Complete Mini; Roche Diagnostics, Mannheim, Germany) at $4{ }^{\circ} \mathrm{C}$ for $30 \mathrm{~min}$. Lysates were cleared by centrifugation at $10,000 \times g$ for $30 \mathrm{~min}$ at $4{ }^{\circ} \mathrm{C}$. Protein concentrations were quantified using the bicinchoninic acid (BCA) method (Pierce BCA Protein Assay Kit; Thermo Fisher Scientific). After dilution in $2 \times$ Laemmli sample buffer (Bio-Rad, Hercules, CA, USA) and incubation at $95^{\circ} \mathrm{C}$ for $5 \mathrm{~min}$, 15 - $\mu$ g protein samples were subjected to electrophoresis on an $8 \%$ SDS-polyacrylamide gel for NBAS and a $12 \%$ SDS-polyacrylamide gel for $\mathrm{p} 31$ and $\beta$-actin and were electroblotted onto polyvinylidene difluoride membranes (Millipore, Burlington, MA, USA). The membranes were blocked with 5\% skimmed milk (Wako Pure Chemical Corporation, Osaka, Japan) in TBST buffer $(1 \times$ Tris-buffered saline, $0.1 \%$ Tween-20) for $1 \mathrm{~h}$ and incubated with primary antibodies (all diluted in $0.5 \%$ skimmed milk) against NBAS (Thermo Fisher Scientific, PA5-49534; 1:1000), p31 (SigmaAldrich, St. Louis, MO, USA, HPA026851; 1:250), and $\beta$-actin (Sigma-Aldrich, A5441; 1:10,000) overnight at $4{ }^{\circ} \mathrm{C}$. Next, the membranes were incubated with horseradish peroxidase (HRP)-conjugated anti-rabbit or anti-mouse secondary antibodies (Jackson ImmunoResearch Laboratories, West Grove, PA, USA; 1:5,000) in TBST, and immunoreactivity was visualized with the Luminate Forte Western HRP substrate (Millipore) according to the manufacturer's protocol, and chemiluminescence signals were digitized using an Amersham Imager 600 (GE Healthcare, Little Chalfont, $\mathrm{UK})$. The $\beta$-actin expression levels of each protein sample served as loading controls. Densitometric measurement of the bands on the Western blots was performed using ImageQuant TL 8.1 1 (GE Healthcare). Data were pooled from three independent experiments. 


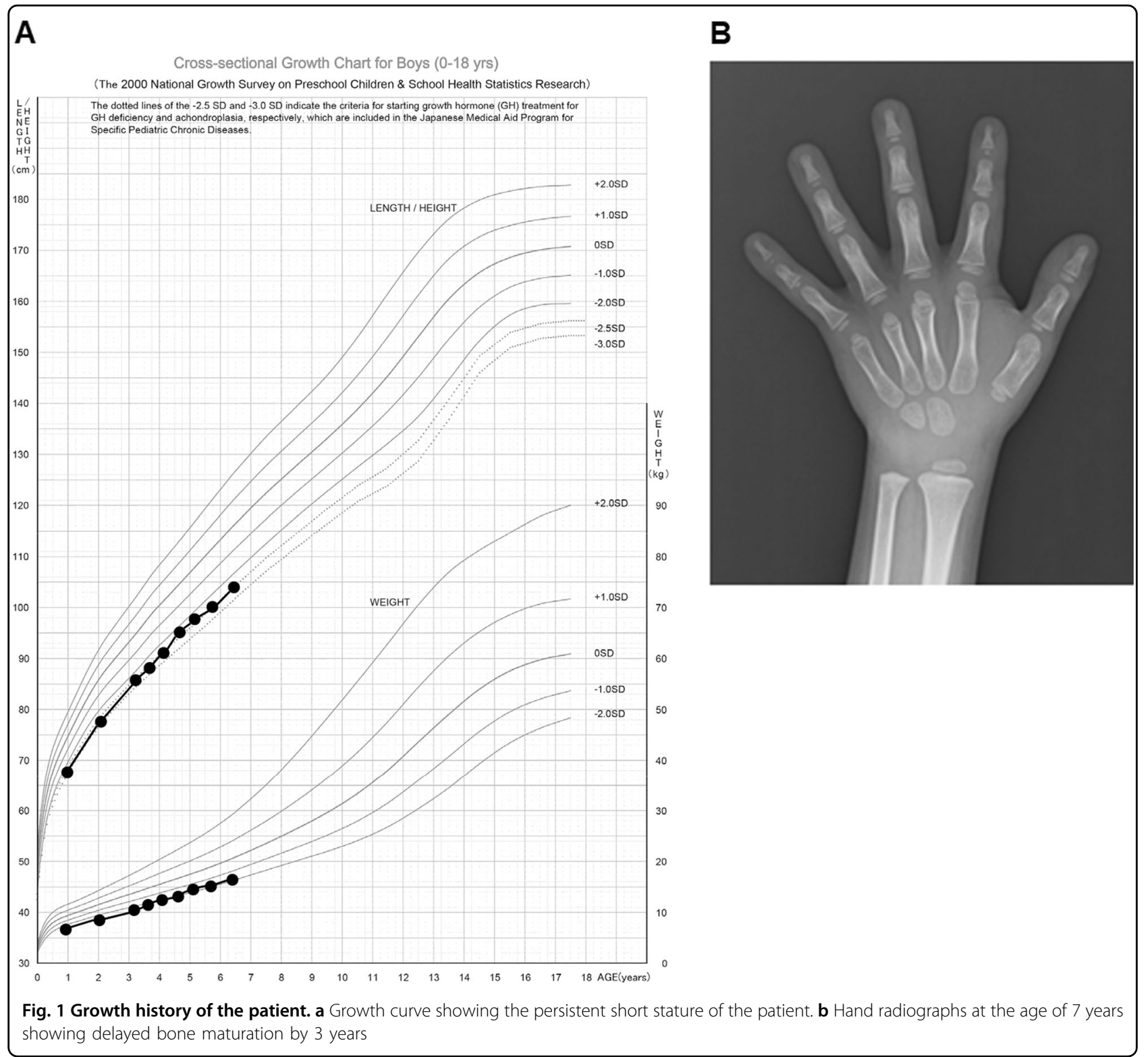

\section{Results}

\section{Patient clinical presentation}

The patient was a Japanese boy born to healthy nonconsanguineous parents at 34 weeks gestation due to a threatened premature delivery. The pregnancy was complicated by fetal growth retardation due to the mother's pregnancy-associated hypertension. The birth weight was $1512 \mathrm{~g}(-2.3 \mathrm{SD})$, and the height was $42.5 \mathrm{~cm}$ $(-0.8 \mathrm{SD})$. During the neonatal period, gallstones were noticed and treated with ursodeoxycholic acid. The patient's psychomotor development was normal. He showed short stature: -2.4 SDs in height at the age of 7 years (Fig. 1a). Hand radiographs revealed delayed bone maturation by 3 years (Fig. 1b). There was no family history of liver disease.
At the age of three years and four months, the patient developed an upper respiratory tract infection with $39^{\circ} \mathrm{C}$ fever, cough, and loss of appetite. On day 5 of his fever, he became lethargic and agitated and was then admitted to our hospital. He had jaundice with an enlarged liver palpable $4 \mathrm{~cm}$ below the costal margin (Fig. 2a). Laboratory results revealed greatly elevated liver enzyme levels (aspartate aminotransferase [AST], 11,023 U/L [normal range: 7-42]; alanine aminotransferase [ALT], $7422 \mathrm{U} / \mathrm{L}$ [normal range: 10-35]; lactate dehydrogenase [LDH], $4968 \mathrm{U} / \mathrm{L}$ [normal range: 120-240]); $\gamma$-glutamyl transpeptidase $[\gamma \mathrm{GT}], 37 \mathrm{U} / \mathrm{L}$ [normal range: 5-60] and cholinesterase [ChE], $239 \mathrm{U} / \mathrm{L}$ [normal range: 222-448]). Total serum bilirubin $(4.4 \mathrm{mg} / \mathrm{dL}$ [normal range: $0.3-1.2])$ and total bile acid $(267.5 \mu \mathrm{mol} / \mathrm{L}$ [normal range: $<10])$ 

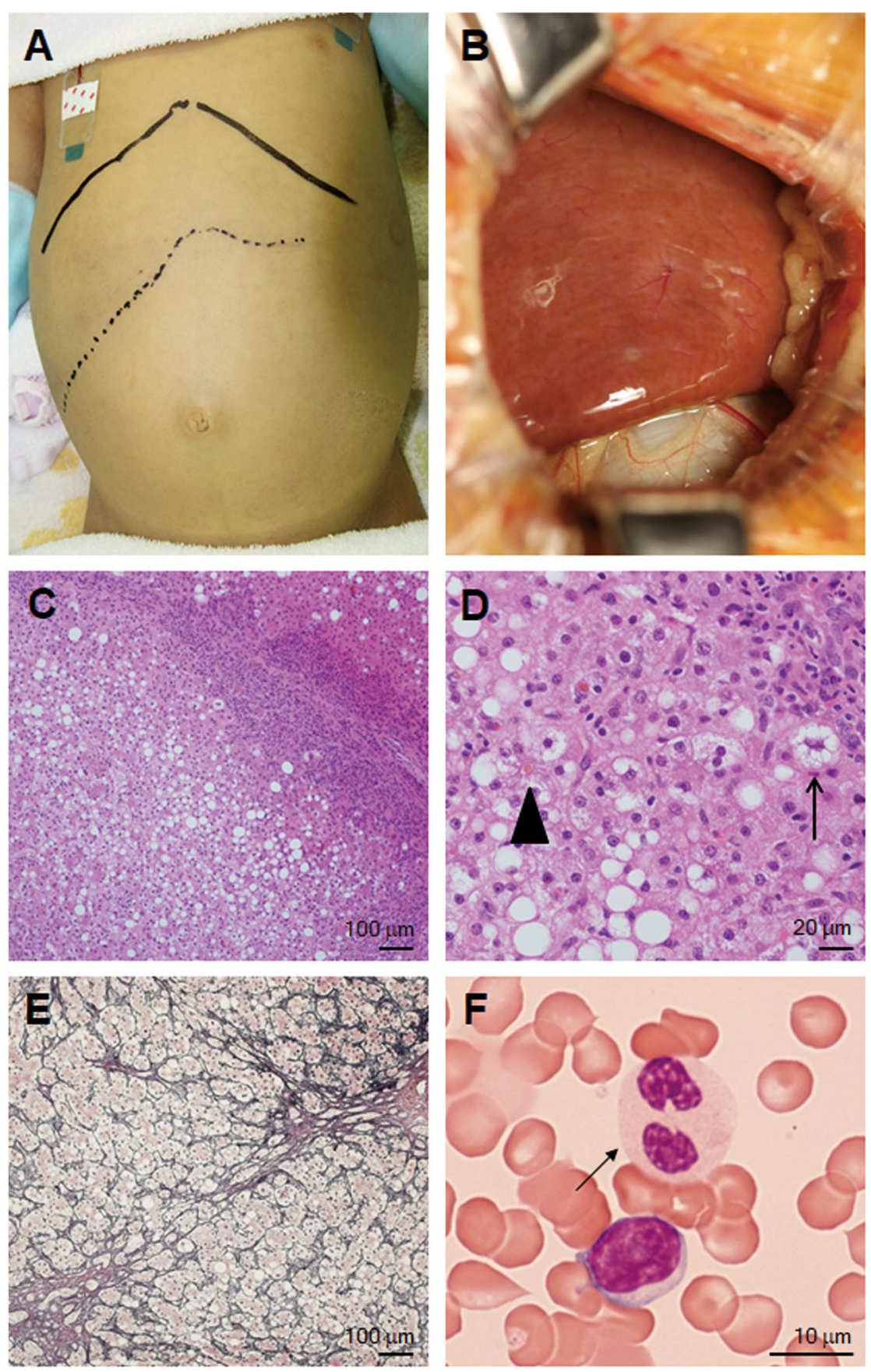

Fig. 2 Abdominal appearance and macroscopic and histopathological findings in the liver and peripheral granulocytes. Photograph of the abdomen showing jaundice and hepatomegaly (a). Macroscopic findings of the liver showed a yellowish appearance and slightly irregular surface (b). Histopathologically, hematoxylin and eosin staining showed moderate parenchymal steatosis and infiltration of inflammatory cells in the portal area (c), microvesicular small lipid droplets in the hepatocytes (arrow), and canalicular bile plug formation (arrowhead) (d). Reticulin staining showed bridging fibrosis (e). Pelger-Huët anomaly in the peripheral granulocytes (arrow) (f)

were significantly increased. Severe coagulopathy (prothrombin time-international normalized ratio (PT-INR), 1.62 [normal range: $0.88-1.12$ ], activated partial thromboplastin time (APTT), > $100 \mathrm{~s}$ [normal range: 26.1-35.8]) was observed. Serum creatinine (Cre) and blood urea nitrogen (BUN) were elevated at $2.83 \mathrm{mg} / \mathrm{dL}$ (normal range: $0.6-1.1$ ) and $66 \mathrm{mg} / \mathrm{dL}$ (normal range: 8-22), respectively, suggesting renal dysfunction. Creatine kinase (CK) was normal (196 mg/dL [normal range: 54-324]). The white blood cell count was elevated and shifted to the 
left, at $17,820 / \mu \mathrm{L}$ (Stabbed 26\%, Segmented $21 \%$ ), and the C-reactive protein (CRP) $(7.15 \mathrm{mg} / \mathrm{dL})$ and serum ferritin (2242 ng/mL [normal range: 30-200]) levels were elevated. His abdominal ultrasound showed severe hepatomegaly without splenomegaly. Electroencephalogram (EEG), magnetic resonance imaging of the brain, electrocardiogram (ECG), and echocardiography were all normal. He was diagnosed as having ALF with renal failure and was managed with intensive treatment, including plasmapheresis, hemodialysis, intravenous antibiotics, intravenous immunoglobulin, and glucose infusion. Following treatment, he made a full recovery and was discharged on the 47th day of disease.

Two months later, at the age of 3 years and 6 months, the patient was readmitted with a second episode of ALF following a sore throat for 2 days and untreated $39^{\circ} \mathrm{C}$ fever. Laboratory tests revealed marked elevation of liver enzymes with coagulopathy and renal failure (AST, 22,291 IU/L; ALT, 7666 IU/L; PT-INR, 5.51; APTT, $73.6 \mathrm{~s}$; Cre, $4.64 \mathrm{mg} / \mathrm{dL}$; BUN, $83 \mathrm{mg} / \mathrm{dL}$; CRP, $7.15 \mathrm{mg} / \mathrm{dL}$; ferritin, $7668 \mathrm{ng} / \mathrm{mL}$ ). Other laboratory data showed changes similar to those of his first ALF episode. Again, complete recovery was achieved following conservative treatment. He was neurologically normal, and there was no impact on his daily life.

Metabolic evaluations during both liver crises showed hyperammonemia $(201 \mu \mathrm{g} / \mathrm{dL}$ [normal range: 12-66]), hypoglycemia $(6 \mathrm{mg} / \mathrm{dL})$, and metabolic acidosis (blood gas analysis [venous]: $\mathrm{pH}$ 7.216, $\mathrm{pCO}_{2} 42.8 \mathrm{mmHg}$, bicarbonate $17.0 \mathrm{mmol} / \mathrm{L}$, base excess $-10.4 \mathrm{mmol} / \mathrm{L}$ ). There was a slight elevation of lactate, at $22.8 \mathrm{mg} / \mathrm{dL}$, but a normal level of pyruvate, $0.67 \mathrm{mg} / \mathrm{dL}$. Serum ketone bodies were elevated (total ketone bodies, $589 \mu \mathrm{mol} / \mathrm{L}$ [normal range: 26-122]; acetoacetate, $371.9 \mu \mathrm{mol} / \mathrm{L}$ [normal range: 13-69]; 3-hydroxybutyrate, $217 \mu \mathrm{mol} / \mathrm{L}$ [normal range: $<76]$ ). Serum amino acid analysis showed elevations in various amino acids, including valine, leucine, methionine, citrulline, phenylalanine, and arginine, suggestive of the secondary effects of substantial liver dysfunction. Free carnitine level was also elevated. Acylcarnitine profiles showed elevations in $\mathrm{C} 0, \mathrm{C} 2, \mathrm{C} 4, \mathrm{C} 5: 1$, C5DC, C6, C10DC, and C12DC. Nonketotic dicarboxylic aciduria was noted in the urinalysis for organic acids, including adipate, suberate, 3-hydroxy-adipate, 3hydroxy-sebacate, dodecanedioic acid, and 3-hydroxydodecanedioic acid. Increased excretion of 2-hydroxy sebacate and tyrosine metabolite $p$-hydroxyphenyllactate (PHPLA) was also noted. All of these changes in metabolic biomarkers normalized between the liver crises. Very long-chain fatty acids in the plasma were normal. Ceruloplasmin, copper, and $\alpha 1$-antitrypsin were all normal. The activities of the mitochondrial respiratory chain complex enzymes I, II, III, and IV were all normal in both the liver tissue and skin fibroblasts, indicating that a mitochondrial disorder was not the etiology of the episodes of ALF.

The workups for the infectious agents Epstein-Barr virus, cytomegalovirus, herpes simplex virus, hepatitis $\mathrm{A}$ virus, hepatitis $B$ virus, and hepatitis $C$ virus were all negative. The immunological workup, including autoantibodies, serum immunoglobulin levels, complement $\mathrm{C} 3$ and $\mathrm{C} 4$ levels, $\mathrm{CD}^{+}{ }^{+}$cell count and function, $\mathrm{CD} 19^{+}$ $B$ cell count, and natural killer cell count, were all unremarkable. The serum cytokine profile at the time of the liver crisis showed the broad elevation of IL-6, neopterin, sTNF-RI, and sTNF-RII, IL-18, but most prominently IL6 and neopterin, suggesting a severe cytokine storm during the crisis. Bone marrow aspiration at the time of the second liver crisis revealed hypercellular marrow with occasional hemophagocytosis with Pelger-Huët anomaly in the granulocytes. A repeated peripheral blood smear showed Pelger-Huët anomaly in approximately 5\% of granulocytes even in the afebrile and asymptomatic states (Fig. 2f).

A liver wedge biopsy was performed shortly after the second ALF crisis. Grossly, the liver showed a yellowish appearance with a slightly irregular surface (Fig. 2b). Histopathologically, the hepatocytes showed patchy degeneration with cytoplasmic vacuolization and moderate macrovesicular steatosis (Fig. 2c). A few microvesicular lipid droplets and bile plug formation were also seen (Fig. 2d). Infiltration of inflammatory cells, mainly $\mathrm{CD}^{+} \mathrm{T}$ cells, was found in the portal area. At the interface of the portal and parenchymal compartments, a ductular reaction was prominent (Fig. 2c). Reticulin staining revealed minimal bridging fibrosis (Fig. 2e). Accumulations of iron and copper were not found by Berlin blue, rhodanine, and rubeanic acid staining (not shown).

\section{Genetic analyses}

Because no etiological diagnosis was reached despite the immunological and metabolic screening, whole-exome sequencing was performed in the patient and in his parents. In total, 25,853 single-nucleotide variants were called. The candidate variations $(n=326)$ were selected by excluding those with (i) ambiguous (unknown) singlenucleotide variations (SNVs) $(n=572)$; (ii) variants only present in unidirectional reads $(n=1702)$; (iii) variant allele frequency $<0.25(n=1593)$ and (iv) known variants listed in SNP databases $(n=21,385)$. From 326 candidate variations, two heterozygous sequence variants in the $N B A S$ gene, chr2:15,629,083C >G (c.1018G>C:p.Gly340Arg) (exon 12) and chr2:15,557,740C >A (c.2674G>T:p.Val892Phe) (exon 24), were detected in the patient. Parental genotyping revealed the c.1018G $>\mathrm{C}$ mutation in the heterozygous state in the father and the c.2674G $>\mathrm{T}$ mutation in the heterozygous state in the mother, indicating compound 


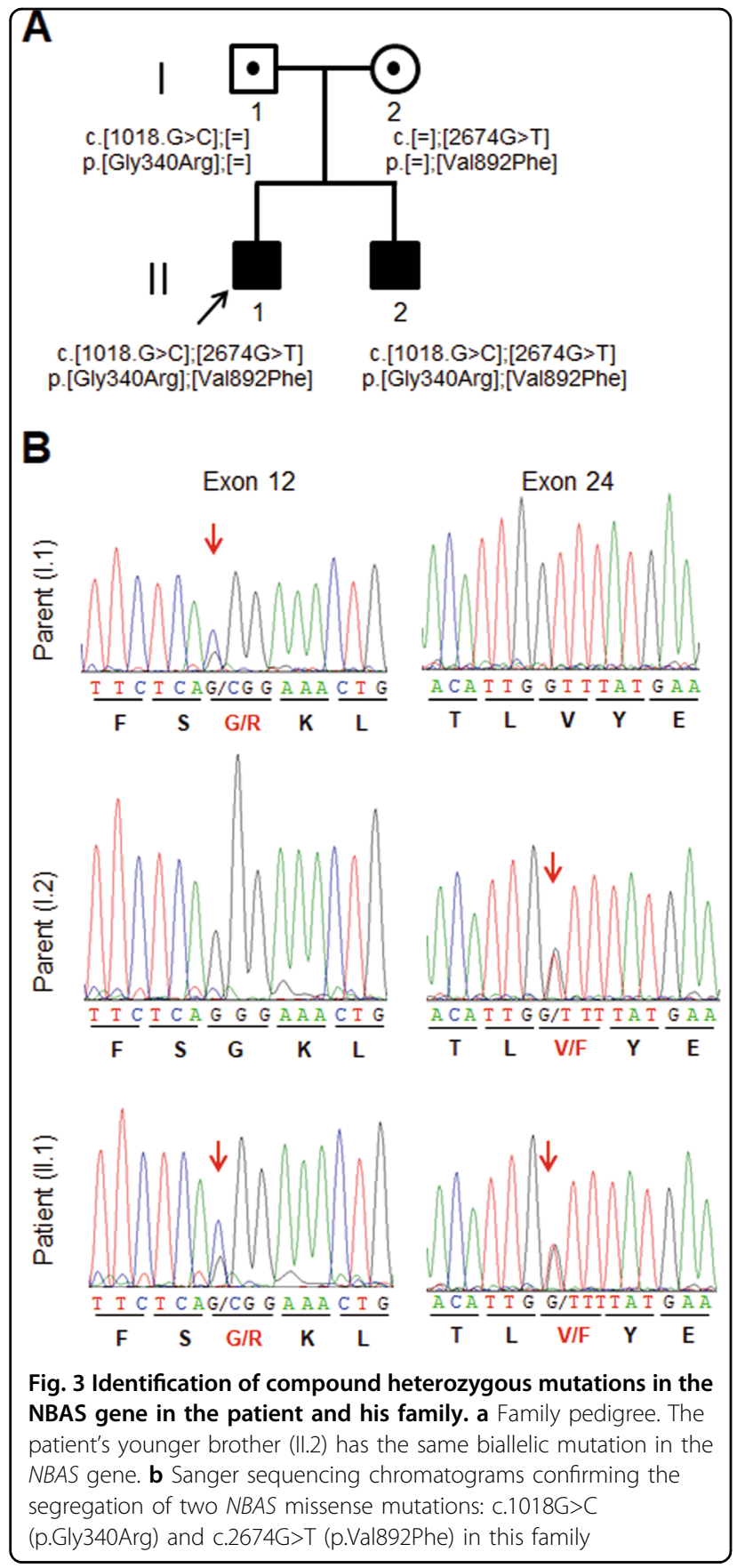

heterozygosity of the NBAS variants in the patient. Both missense variants were validated by Sanger sequencing (Fig. 3). Neither mutation was present in $>120,000$ alleles from the Exome Aggregation Consortium (ExAC) Server, and they affect highly conserved amino acid residues. In iJGVD, the largest Japanese genetic variation database ${ }^{20}$, chr2:15,557,740C >A (c.2674G>T:p.Val892Phe) was not identified, and the allele frequency of chr2:15,629,083C $>\mathrm{G}$ (c.1018G>C:p.Gly340Arg) was $0.0001(n=1 / 7,102)$.

In silico analyses predicted these variants to be functionally relevant (PolyPhen 2: probably damaging [1.000];
Provean: deleterious [-7.035 (p.Gly340Arg), -3.838 (p.Val892Phe)]; MutationTaster: disease-causing). Neither NBAS mutation had been previously reported in cases of ILFS type 2 or SOPH syndrome. Genetic diagnosis was carried out on the patient's 2-year-old younger brother to evaluate the possibility of a presymptomatic state of the $N B A S$ mutation-based disease. He was identified as carrying the biallelic mutations in NBAS (Figs. 3, 4).

\section{Western blot analysis of NBAS and p31}

The NBAS protein levels in the fibroblast sample from the patient were not decreased, even after changing the culturing temperature from 37 to $40{ }^{\circ} \mathrm{C}$; however, p31 protein levels were significantly reduced (Fig. 5). As p31 forms the SNARE complex with NBAS, mutations in $N B A S$ may impair NBAS-p31 interactions, resulting in a loss of protein stability of p31 followed by dysregulation of Golgi-to-endoplasmic reticulum (ER) retrograde vesicular trafficking in the patient.

\section{Discussion}

NBAS mutations were first identified in SOPH syndrome patients, but they do not commonly exhibit a liver phenotype ${ }^{5}$. In 2015, Haack et al. ${ }^{4}$ identified homozygous or compound heterozygous NBAS gene mutations in German patients with recurrent ALF. More than 15 existing studies on cases carrying homozygous or compound heterozygous $N B A S$ gene mutations revealed the broad phenotypic spectrum of $N B A S$ mutation-based diseases, ranging from isolated recurrent ALF to a multisystemic disease with short stature, skeletal dysplasia, facial dysmorphism, immunological abnormalities, Pelger-Huët anomaly, and optic nerve atrophy resembling SOPH syndrome (Fig. 4) ${ }^{4-18}$. The extrahepatic phenotypes observed in our patient were Pelger-Huët anomaly and short stature. He did not present with optic atrophy, facial dysmorphism, or skeletal dysplasia.

The liver phenotype in NBAS mutation-based disease is most typically recurrent ALF triggered by febrile infections, but mild elevations of liver biochemical values occur in some cases ${ }^{4,6-14,16,17}$. These elevations often develop during infancy or early childhood and become less frequent with age. Coagulopathy and jaundice were observed in most of the cases. Secondary metabolic abnormalities, including hypoglycemia and hyperammonemia, were often observed. Complete recovery is typically achieved with antipyretic therapy and induction of anabolism, including parenteral glucose and lipids. Our patient developed ALF twice at the age of 3 years, both episodes triggered by febrile infections. He showed severe coagulopathy with renal failure but recovered completely with conservative treatment. After the diagnosis of $N B A S$ mutation-based disease, he suffered multiple hospitalizations with febrile illnesses associated with liver 


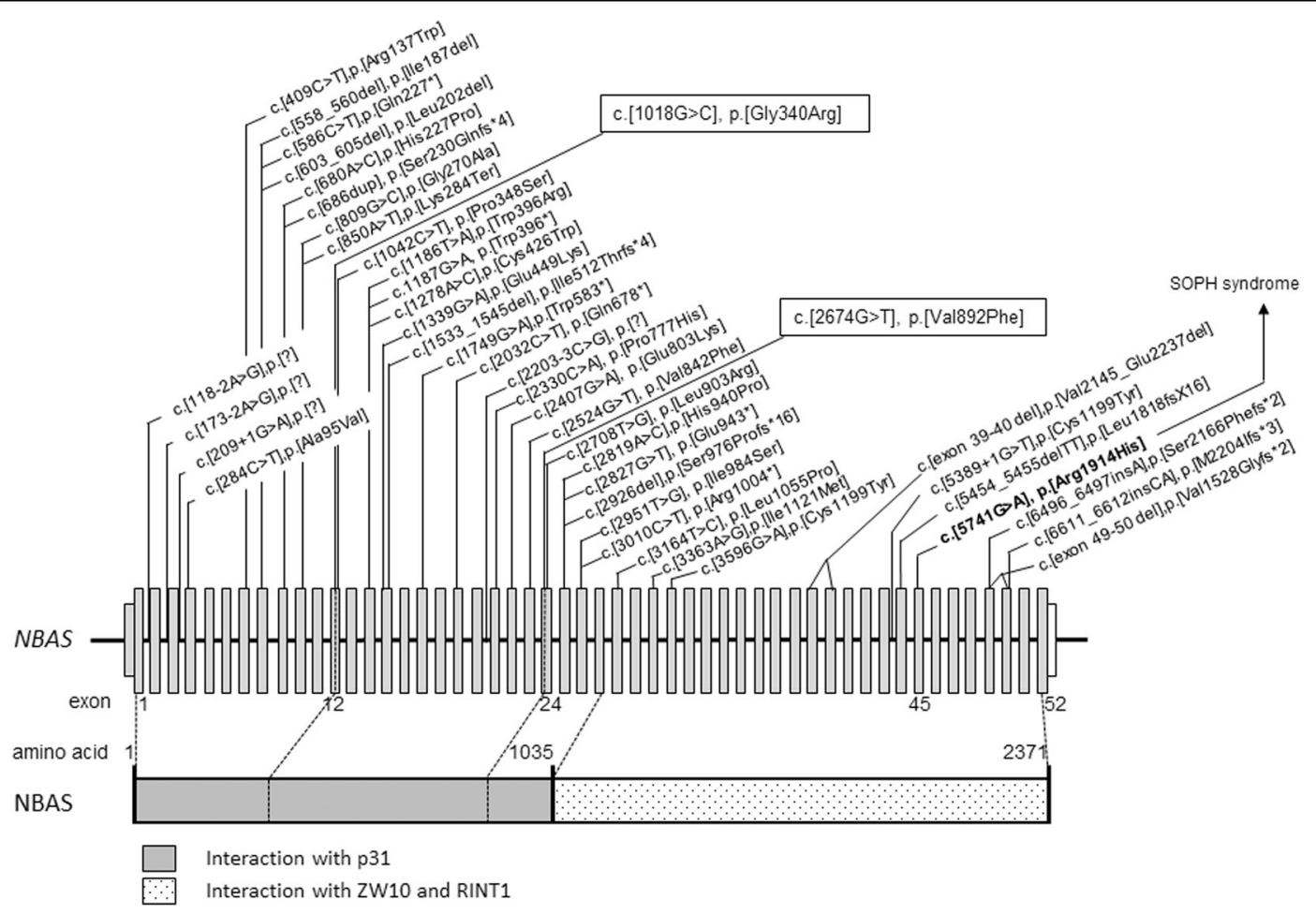

Fig. 4 Gene and protein structures of NBAS and identified mutations. Schematic gene and protein structures of NBAS showing the p31 interaction site at the N-terminus and the ZW10 and RINT1 interaction site at the C-terminus. Mutations associated with acute liver failure (ALF), including those in our patient, were mapped to the N-terminal end, whereas mutations associated with SOPH syndrome or related diseases without the liver phenotype were mapped near the C-terminal end. Mutations identified in this study are boxed

dysfunction; however, early antipyretic therapy using intravenous injection of acetaminophen and glucose infusion prevented further occurrences of ALF until the age of seven. His younger brother was found to carry the same mutations as the proband's at the age of 2 years. He shared only Pelger-Huët anomaly in peripheral granulocytes as a symptom of NBAS mutation-based disease. After the genetic diagnosis, he has received early antipyretic therapy and has never experienced fever-triggered ALF or liver dysfunction to date (he is currently 5 years old). These findings indicate the possible effectiveness of early antipyretic therapy to prevent life-threatening ALF. However, it is also possible that there is a phenotypic heterogeneity of the affected organs among the family members. Further investigation to reveal the penetration and phenotype-genotype correlation in NBAS mutationbased disease is awaited.

The molecular pathogenesis by which NBAS contributes to liver disease and to fever dependency is not fully understood. The NBAS protein is considered to be involved in Golgi-to-ER retrograde vesicular trafficking and to control nonsense-mediated mRNA decay ${ }^{21-23}$. The NBAS protein interacts directly with p31, ZW10, and RINT1 and is believed to play a primary role in SNARE assembly at the $\mathrm{ER}^{21}$. It is proposed that the site at amino acid positions $1-1035$ is the p31 binding site. Mutations associated with ALF, including those in our patient, are mostly found at the N-terminal end of NBAS (Fig. 4). In contrast, the p.Arg1914His mutation carried by SOPHaffected Yakut individuals in the homozygous state is located near the C-terminal end and does not cause ALF ${ }^{5}$. Our patient's skin fibroblasts showed a decreased p31 protein level (Fig. 5). Thermal susceptibilities of NBAS and $\mathrm{p} 31$ proteins have been reported previously ${ }^{4,8}$. These findings suggest that the loss of the NBAS-p31 interaction may cause alterations to Golgi-to-ER retrograde vesicular trafficking, which leads to liver dysfunction, especially in the febrile condition ${ }^{8}$. Further in-depth mechanistic studies are needed to establish the genotype-phenotype correlations and to reveal the wide phenotypic spectrum of NBAS mutation-based disease. Further investigations using the patient's iPS cell-derived hepatocytes will show whether the episodes of liver disease are due to alterations in Golgi-to-ER retrograde vesicular trafficking and/or impaired nonsense-mediated mRNA decay, or a yet-tobe-discovered NBAS function.

We describe the first reported Japanese case of fevertriggered recurrent ALF carrying novel compound heterozygous missense mutations of $N B A S$. We recommend sequencing $N B A S$ in any patients with unexplained 


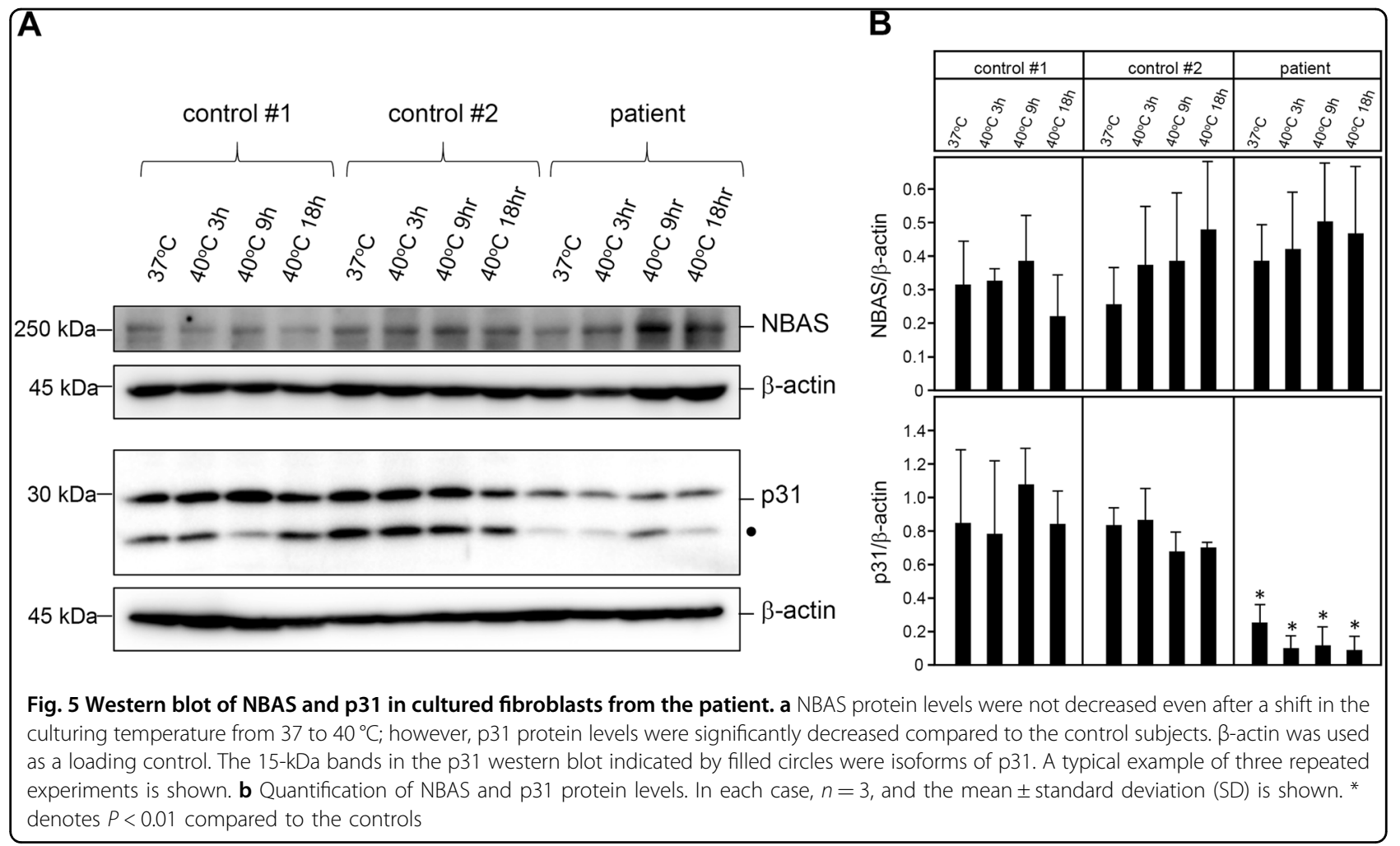

recurrent ALF, especially in individuals with feverassociated hepatic dysfunction.

\section{Acknowledgements}

We are grateful to the patient's family, who contributed to this study. We thank Dr. Kei Murayama at the Department of Metabolism, Chiba Children's Hospital, for the mitochondrial respiratory chain complex enzyme activities analyses and Dr. Taizo Wada at the Department of Pediatrics, School of Medicine, Kanazawa University, for the serum cytokine profile analysis. We also thank Ms. Ayaka Ono, Ms. Yuko Kanatani, Mr. Yusuke Okuno, and Mr. Hideki Muramatsu for their technical assistance in conducting this study. This work was supported in part by a grant from the Practical Research for Innovative Cancer Control from the Japan Agency for Medical Research and Development

(AMED_15ck0106066h0002) to Y.H. and from the Japan Society for the Promotion of Science (JSPS) Grant-in-Aid for Challenging Exploratory Research (15K14465) to J.M.

\section{Author details}

'Department of Pediatrics, Kawasaki Medical School, Okayama 701-0192, Japan ${ }^{2}$ Department of Pathophysiology and Metabolism, Kawasaki Medical School, Okayama 701-0192, Japan. ${ }^{3}$ Department of Pathology, Kawasaki Medical School, Okayama 701-0192, Japan. ${ }^{4}$ Department of Pediatrics and Child Health, Kurume University School of Medicine, Kurume 830-0011, Japan. ${ }^{5}$ Department of Pathology, Kurume University School of Medicine, Kurume 830-0011, Japan. ${ }^{6}$ Research Center for Innovative Cancer Therapy, Kurume University School of Medicine, Kurume 830-0011, Japan. ${ }^{7}$ Department of Pathology and Tumor Biology, Graduate School of Medicine, Kyoto University, Kyoto 606-8501, Japan. ${ }^{8}$ Laboratory of DNA Information Analysis, Human Genome Center, Institute of Medical Science, The University of Tokyo, Tokyo 108-8639, Japan. ' ${ }^{9}$ Laboratory of Sequence Analysis, Human Genome Center, Institute of Medical Science, The University of Tokyo, Tokyo 108-8639, Japan. ${ }^{10}$ Institute of Physiology and Medicine, Jobu University, Gunma 370-1393, Japan. ${ }^{11}$ Department of Child Health and Development, Graduate School of Medical and Dental Sciences, Tokyo Medical and Dental University (TMDU), Bunkyo-ku, Tokyo 113-8519, Japan

\section{Conflict of interest}

The authors declare that they have no conflict of interest.

\section{Publisher's note}

Springer Nature remains neutral with regard to jurisdictional claims in published maps and institutional affiliations.

Received: 4 October 2018 Revised: 13 November 2018 Accepted: 16 November 2018.

Published online: 7 January 2019

\section{References}

1. Squires, R. H. et al. Acute liver failure in children: the first 348 patients in the pediatric acute liver failure study group. J. Pediatr. 148, 652-658 (2006).

2. Narkewicz, M. R. et al. Pediatric Acute Liver Failure Study Group, Pattern of diagnostic evaluation for the causes of pediatric acute liver failure: an opportunity for quality improvement. J. Pediatr. 155, 801-806 (2009).

3. D'Agostino, D., Diaz, S., Sanchez, M. C. \& Boldrini, G. Management and prognosis of acute liver failure in children. Curr. Gastroenterol. Rep. 14, 262-269 (2012).

4. Haack, T. B. et al. Biallelic mutations in NBAS cause recurrent acute liver failure with onset in infancy. Am. J. Hum. Genet. 97, 163-169 (2015).

5. Maksimova, N. et al. Neuroblastoma amplified sequence gene is associated with a novel short stature syndrome characterised by optic nerve atrophy and Pelger-Huët anomaly. J. Med. Genet. 47, 538-548 (2010).

6. Capo-Chichi, J. M. et al. Neuroblastoma amplified sequence (NBAS) mutation in recurrent acute liver failure: confirmatory report in a sibship with very early onset, osteoporosis and developmental delay. Eur. J. Med. Genet. 58, 637-641 (2015).

7. Segarra, N. G. et al. NBAS mutations cause a multisystem disorder involving bone, connective tissue, liver, immune system, and retina. Am. J. Med. Genet. A. 167A, 2902-2912 (2015) 
8. Staufner, C. et al. Recurrent acute liver failure due to NBAS deficiency: phenotypic spectrum, disease mechanisms, and therapeutic concepts. J. Inherit Metab. Dis. 39, 3-16 (2016).

9. Kortüm, F. et al. Acute liver failure meets SOPH syndrome: a case report on an intermediate phenotype. Pediatrics 139, e20160550 (2017).

10. Balasubramanian, M. et al. Compound heterozygous variants in NBAS as a cause of atypical osteogenesis imperfecta. Bone 94, 65-74 (2017).

11. Calvo, P. L. et al. NBAS mutations cause acute liver failure: when acetaminophen is not a culprit. Ital. J. Pediatr. 3, 88 (2017).

12. Li, J. Q. et al. Novel NBAS mutations and fever-related recurrent acute liver failure in Chinese children: a retrospective study. Bmc. Gastroenterol. 17, 77 (2017).

13. Hasosah, M. Y. et al. Neuroblastoma amplified sequence gene mutation: a rare cause of recurrent liver failure in children. Saudi J. Gastroenterol. 23, 206-208 (2017).

14. Regateiro, F. S. et al. Recurrent elevated liver transaminases and acute liver failure in two siblings with novel bi-allelic mutations of NBAS. Eur. J. Med. Genet. 60, 426-432 (2017).

15. Park J. W., Lee S. J. Foveal hypoplasia in short stature with optic atrophy and Pelger-Huët anomaly syndrome with neuroblastoma-amplified sequence (NBAS) gene mutation. J. AAPOS 2017; pii: S1091-8531(17)30034-4
16. Palagano, E. et al. Mutations in the Neuroblastoma Amplified Sequence gene in a family affected by Acrofrontofacionasal Dysostosis type 1. Bone $\mathbf{1 1 4}$ 125-136 (2018).

17. Wang, J., Pu, Z. \& Lu, Z. Targeted next-generation sequencing reveals two novel mutations of NBAS in a patient with infantile liver failure syndrome-2. Mol. Med. Rep. 17, 2245-2250 (2018).

18. Sunwoo, Y., Kim, Y. M., Kim, E. N., Oh, S. H. \& Lee, B. H. Severe form of neuroblastoma amplified sequence deficiency in an infant with recurrent acute liver failure. Pediatr. Int. 60, 302-304 (2018).

19. Kunishima, S. et al. ACTN1 mutations cause congenital macrothrombocytopenia. Am. J. Hum. Genet. 92, 431-438 (2013).

20. Nagasaki M. et al. Rare variant discovery by deep whole-genome sequencing of 1,070 Japanese individuals. Nat. Commun. 6, 8018 (2015)

21. Aoki, T. et al. Identification of the neuroblastoma-amplified gene product as a component of the syntaxin 18 complex implicated in Golgi-to-endoplasmic reticulum retrograde transport. Mol. Biol. Cell. 20, 2639-2649 (2009).

22. Longman, D. et al. DHX34 and NBAS form part of an autoregulatory NMD circuit that regulates endogenous RNA targets in human cells, zebrafish and Caenorhabditis elegans. Nucleic Acids Res. 41, 8319-8331 (2013).

23. Hug, N., Longman, D. \& Cáceres, J. F. Mechanism and regulation of the nonsense-mediated decay pathway. Nucleic Acids Res. 44, 1483-1495 (2016). 